Ann. Zootech., I962, 11 (I), 39-5I.

\title{
ÉVOLUTION DE L'ANABOLISME GRAVIDIQUE CHEZ LA TRUIE EN FONCTION DE L'ÂGE DE L'ANIMAL
}

\author{
P. ROMBAUTS \\ Avec la collaboration technique de Ginette Léprine, Françoise Thuret et J. M. Boisseau. \\ Station de Biochimie et de Nutrition, Centre national de Recherches zootechniques, \\ Jouy-en-Josas (Seine-et-Oise).
}

SOMMAIRE:

Le métabolisme de l'azote est étudié chez la Truie pendant la gestation et comparé à celui d'aninaux de même âge et même poids au départ, mais non gravides. Comme la croissance se poursuit longtemps après la puberté dans l'espèce porcine on ne peut parler d'anabolisme " maternel " de gestation qu'en défalcuant les rétentions dues à la croissance. Cet anabolisme gravidique ne se manifeste qu'en fin de croissance et surtout chez l'adulte.

L'anabol'sme apparaît dès le début de la gestation mais s'accentue nettement à partir de la $\mathrm{II}^{\mathrm{e}}$ semaine. Les capacités d'utilisation alimentaire sont remarquablement élevées : les coefficients de rétention de l'azote sont voisins de $30 \mathrm{p}$. Ioo pendant la $2^{\mathrm{e}}$ moitié de la gestation, même chez les animaux âgés ; ce qui correspond, exprimé en tissu musculaire, à des gains corporels d'environ $30 \mathrm{~kg}$ pour la mère et ceci malgré un niveau alimentaire modeste.

\section{INTRODUCTION}

Depuis les travaux d'Evans (I929) on sait que la Truie présente des rétentions élevées au cours de la gestation, et, depuis ceux de Mirchel, et al. (I93I), on connait avec précision les quantités d'éléments azotés et minéraux déposés dans l'utérus gravide. Mais ce n'est que ces dernières années que l'on a étudié l'anabolisme maternel de la Truie soit par la méthode des bilans (LENkEIT et $a l$., I955, I956) soit par l'examen des gains de poids des tissus de femelles gestantes (SALMon-I_EGAGNEur et JACQUOT, I96I a).

Nos recherches antérieures chez la Ratte (ROMBAUTs, BOURDEr, et JACQUOT, I956) et la Brebis (RombauTs I959 $a$ et $c$ ) nous ont montré que si l'anabolisme se manifestait même dans des conditions nutritionnelles défavorables, l'ampleur des rétentions restait toutefois dépendante du niveau de nutrition énergique et azoté. 
Ainsi, Boyne, Chalmers et Cuthbertson (1953) chez le Rat ont obtenu des rétentions d'azote ne correspondant qu'aux seuls organes reproducteurs. Alors que, dans leurs expériences sur la Truie LENkEIT et al. (I956) ont montré que la gain d'azote maternel - contenu utérin déduit - pouvait varier dans des proportions telles, qu'exprimé en tissu musculaire - il représentait des gains allant de I7 à 53 kilos suivant la valeur énergétique et azotée des rations allouées en cours de gestation.

Mais pour mesurer avec rigueur l'anabolisme maternel de gestation, c'est-à-dire les rétentions d'azote ou de minéraux provoquées par la gravidité dans le corps de la mère, en plus des éléments fixés dans 1'utérus et les mamelles, il est nécessaire de connaître le métabolisme d'animaux ṇon gravides de même âge et même poids soumis aux mêmes conditions expérimentales, notamment alimentaires.

Cette nécessité est particulièrement impérieuse lorsqu'on étudie une espèce comme les porcins où la croissance se poursuit longtemps après la puberté (L,oDGE, Mc Donal, et Mc Pherson r96I). L'examen des courbes de poids des animaux nourris ad libitum, (SALMON et JACQUOT I96I $b$ ) est significatif à cet égard : lorsque les truies gravides ont pris $80 \mathrm{~kg}$ pendant la gestation, les animaux témoins ont gagné $40 \mathrm{~kg}$ pendant le même temps.

Dans le dessein d'étudier ultérieurement le déterminisme hormonal des facultés anabolisantes de la gestation chez la 'Truie, nous avons donc mesuré les bilans d'azote de truies gestantes et d'animaux non gravides, le métabolisme de ceux-ci représentant les rétentions permises par le niveau nutritionnel adopté et celles liées, compte tenu de leur âge, à l'entretien et à la croissance. Comme nous avons constaté dans nos premiers essais qu'il n'y avait pas de véritable anabolisme gravidique quand la crois sance était encore très active, nous avons répété nos expériences sur des animaux plus âgés.

\section{MÉTHODES EXPÉRIMENTALES}

Les expériences da bilan ont été effectuées avec des truies de race Large White de même provenance, mềne âge et même poids. Au début de l'expérience I, l'âge de l'ensemble de nos animaux était de 3,37 jours animaux), et l'expérience III sur 6 animitux. Nous avons conservé . il un animal près . - les mêmes témoins dans les 3 expériences successives afin d'obtenir une meilleure précision dans l'appréciation de la croissance.

Fn ce qui concerne, l'expérience I, nous ne rapportons que les résultats du début de la gestation, les truies gravides ayant subi par la suite des interventions chirurgicales d'ablation de fixtus et de corps jaunes. Au contraire, dans les expériences II et III, nous n'avons placé nos animaux en cage à métabolisme qu'à partir de la $6^{\mathrm{e}}$ semaine de gestation, la deuxième moitié de la gestation étant la période d'anabolisme la plus active et ceci nous permettant de contrôler au début la réussite de la saillie en observant l'absence des chaleurs.

Le régime utilisé au cours des trois expériences présentait la composition centésimale suivante:

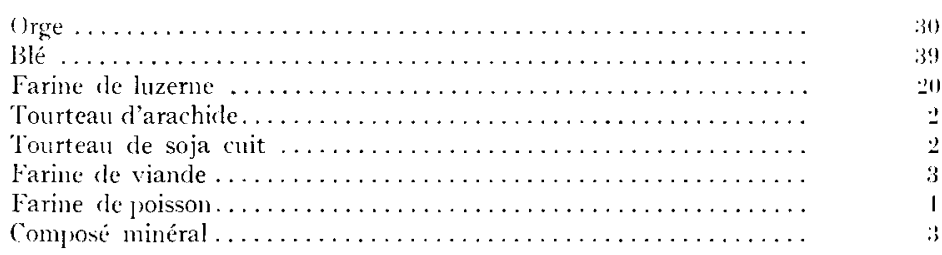


A ce régime on a ajouté les quantités de vitamines suivantes, à I00 kg d'aliment:500000 UI de vitamine $\mathrm{A}, 50000 \mathrm{UI}$ de vitamine $\mathrm{D}_{3}$, ro g de choline, $200 \mathrm{mg}$ de riboflavine, $500 \mathrm{mg}$ de pantothénate de calcium et I mo de vitamine $B_{\cdot 12}$

Le composé minéral contenait, en pourcentage : $\mathrm{CaIPO}_{4} \cdot{ }_{2} \mathrm{H}_{2} \mathrm{O}: 32,567 ; \mathrm{NaCl}: 23,800 ; \mathrm{KCl}$; I 2, $400 ; \mathrm{MgCl}_{2}:$ I 5,$000 ; \mathrm{KH}_{2} \mathrm{PO}_{4}:$ I0,I00; Fe SO$\cdot 7 \mathrm{H}_{2} \mathrm{O}: 5,470 ; \mathrm{Mn} \mathrm{SO}_{4}: 0,062 ; \mathrm{CUSO}_{4}: 0,035:$ $\mathrm{ZnSO}_{4}: 0,100 ; \mathrm{CoSO}_{4}: 0,066 ; \mathrm{Kl}: 0,400$.

L'analyse chimique a donné la composition suivante, en p. 100 d'aliment brut :

\begin{tabular}{|c|c|}
\hline Latières & $\ldots \ldots \ldots$ \\
\hline - & 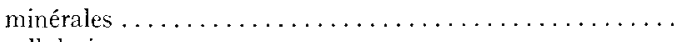 \\
\hline - & 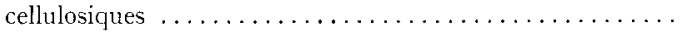 \\
\hline - & azotées $\ldots \ldots \ldots \ldots \ldots \ldots \ldots \ldots \ldots \ldots \ldots \ldots \ldots \ldots \ldots$ \\
\hline - & $\cdots \ldots \ldots \ldots \ldots \ldots \ldots \ldots \ldots \ldots \ldots \ldots \ldots \ldots \ldots$ \\
\hline
\end{tabular}

Le régime était donné sous forme de pâtée, à raison d'une partic de farine pour 2 parties d'eau, en 2 repas journaliers.

Pendant les expériences I et III, tous les animaux ont reçu la même quantité d'aliment soit $2,5 \mathrm{kgs}$ d'aliment sec par jour, fournissant $60 \mathrm{~g}$ d'azote. Au cours de l'expérience II, une augmentation des quántités ingérées en fin de gestation a porté cet apport journalier à $77,5 \mathrm{~g}$ d’azote en moyenne.

Les truies ont été maintenues de façon continue pendant toute la durée des expériences dans des cages à métabolisme construites spécialement pour cette espèce et dont nous donnerons la description dans une publication ultérieure. Pour éviter toute perte d'azote, les urines et les fèces étaient cecueillies sur acide chlorhydrique et les dosages d'azote (Kjeldahl) effectués sur fèces fraîches.

\section{RÉSULTATS}

La cinétique des rétentions au cours de la gestation apparaît clairement par une représentation graphique (fig. I à 6). Le régime alimentaire étant le même pour tous les animaux et la gestation n'entraînant pas de modification de la digestibilité apparente des protéines, nous n'avons fait figurer sur ces courbes ni l'azote ingéré, ni l'azote fécal, mais directement l'azote absorbé. La différence d'ordonnée avec l'azote urinaire donne donc en chaque point l'azote retenu.

Dans les tableaux ( $I$, 2 et 3 ) sont résumés les résultats des périodes expérimentales concernant les données pondérales ainsi que les coefficients de digestibilité et les coefficients de rétention correspondants. Sauf la truie saillie à 7 I 7 jours, toutes les mesures portent sur la $I^{\text {re }}$ gestation de nos animaux. Il ne peut donc y avoir d'interférence d'une gestation et d'une lactation antérieure. On constate déjà un anabolisme. gravidique pendant les six premières semaines de gestation. L'excédent de rétention des gestantes par rapport aux témoins est de $157,2 \mathrm{~g}$ d'azote, soit 30,3 p. Ioo d'augmentation (tableau. I). Mais ce résultat a été obtenu sur des animaux jetnnes, à croissance encore active. La différence serait probablement plus importante chez des adultes.

En effet, la comparaison des résultats des expériences II et III nous montrent que si les rétentions en fin de gestation varient peu avec 1'âge des animaux, celle des témoins diminuant, l'écart entre animaux gravides et témoins s'accentue jusqu'à l'âge adulte (tableaux 2 et 3 ). Chez les animaux saillis à 490 jours, les truies gravides retiennent $\mathrm{I} 2,7 \mathrm{~g}$ d'azote par jour alors que les témoins n'en retiennent que $5, \mathrm{r} \mathrm{g}$. 


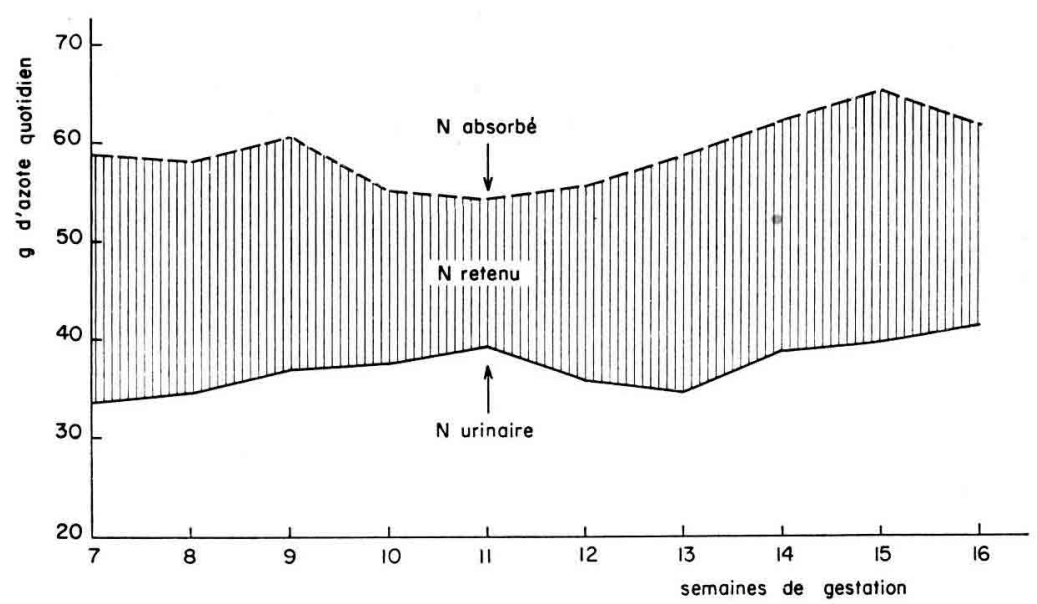

Fig. I. - Expérience II - Bilan d'azote des T'ruies gravides

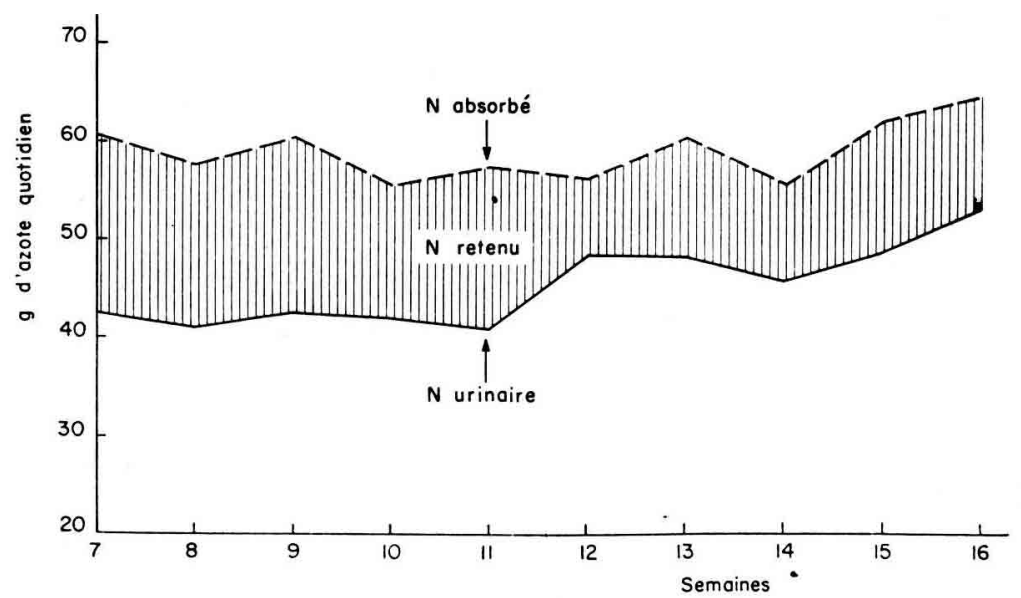

FIG. 2. - Expérience $I I-$ Bilan d'asote des Truies témoins

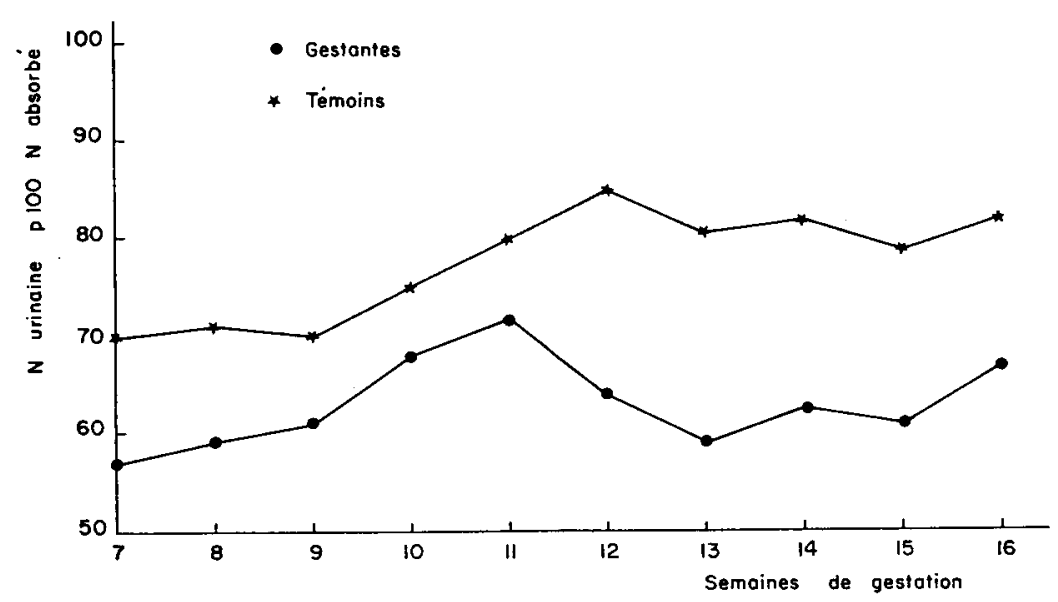

FIc. 5. - Expérience $I I-$ Cinétiqu de lexcrétion urinaire d'asote 


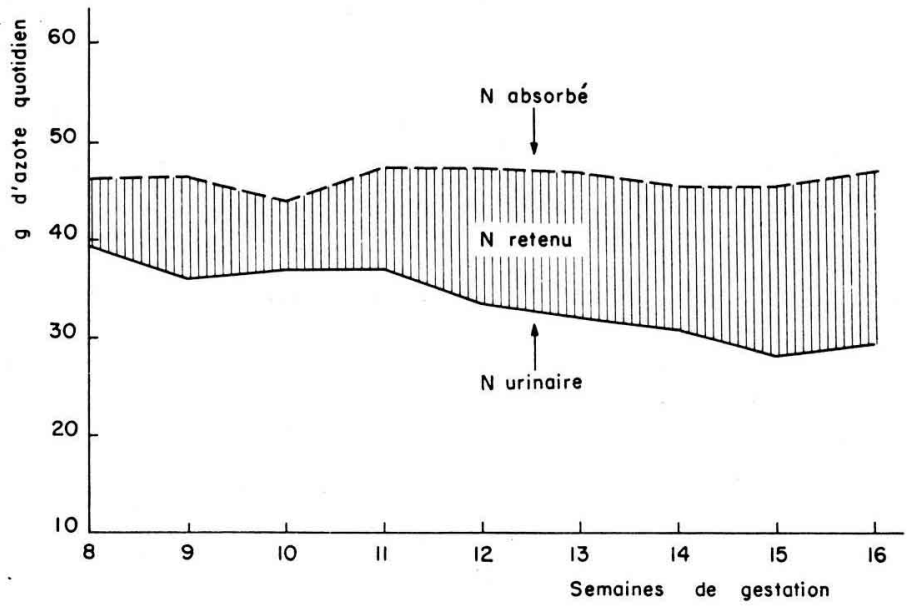

FIG. 3. - Expérience III - Bilan d'azote des Truies gravides

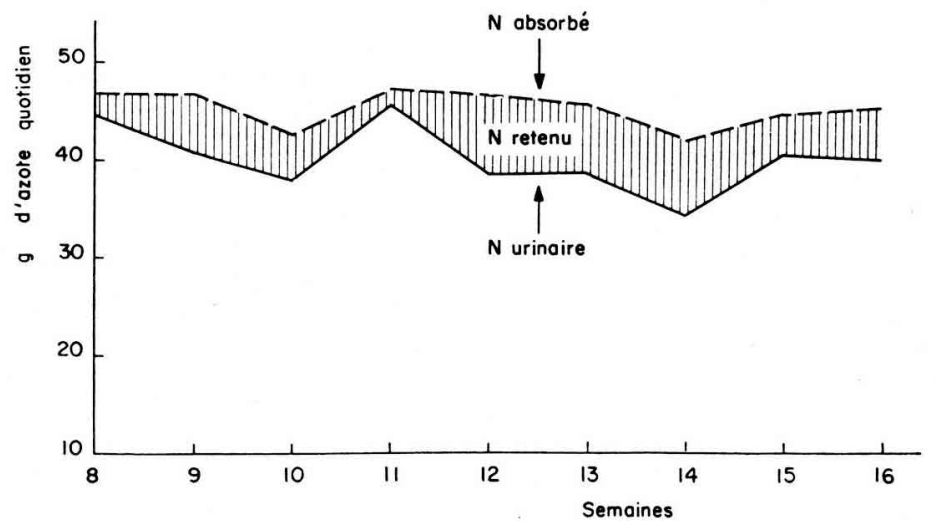

FIG. 4. - Expérience III - Bilan d'azote des Truies témoins

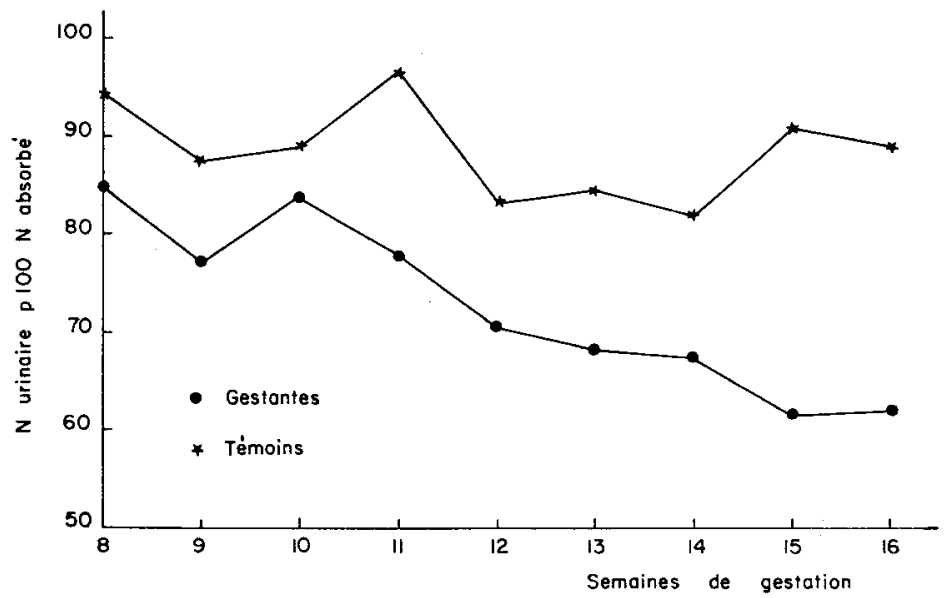

FIG. 6. - Expérience III - Cinétique de l'excrétion urinaire d'azote 
P. ROMBAUTS

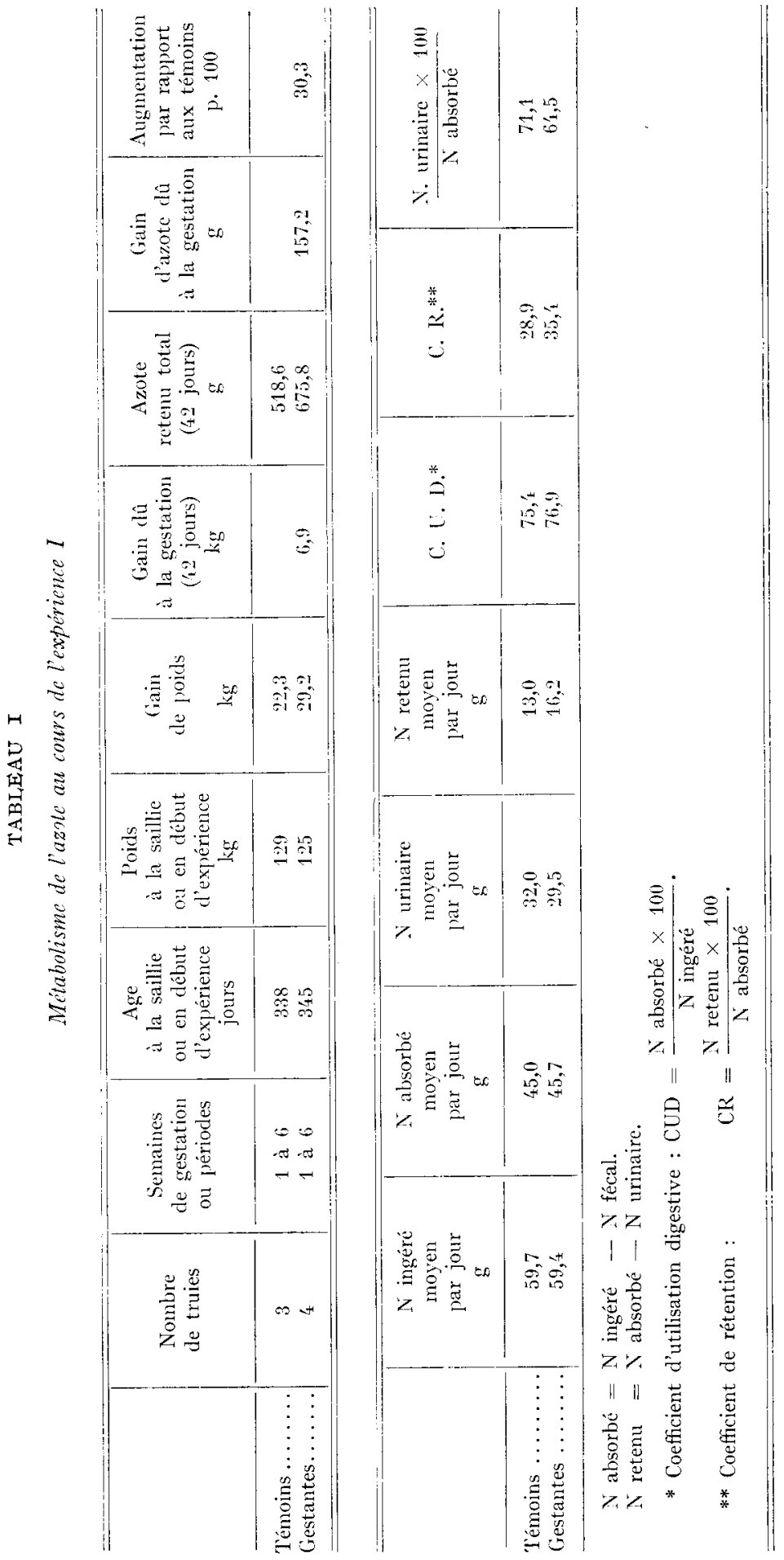




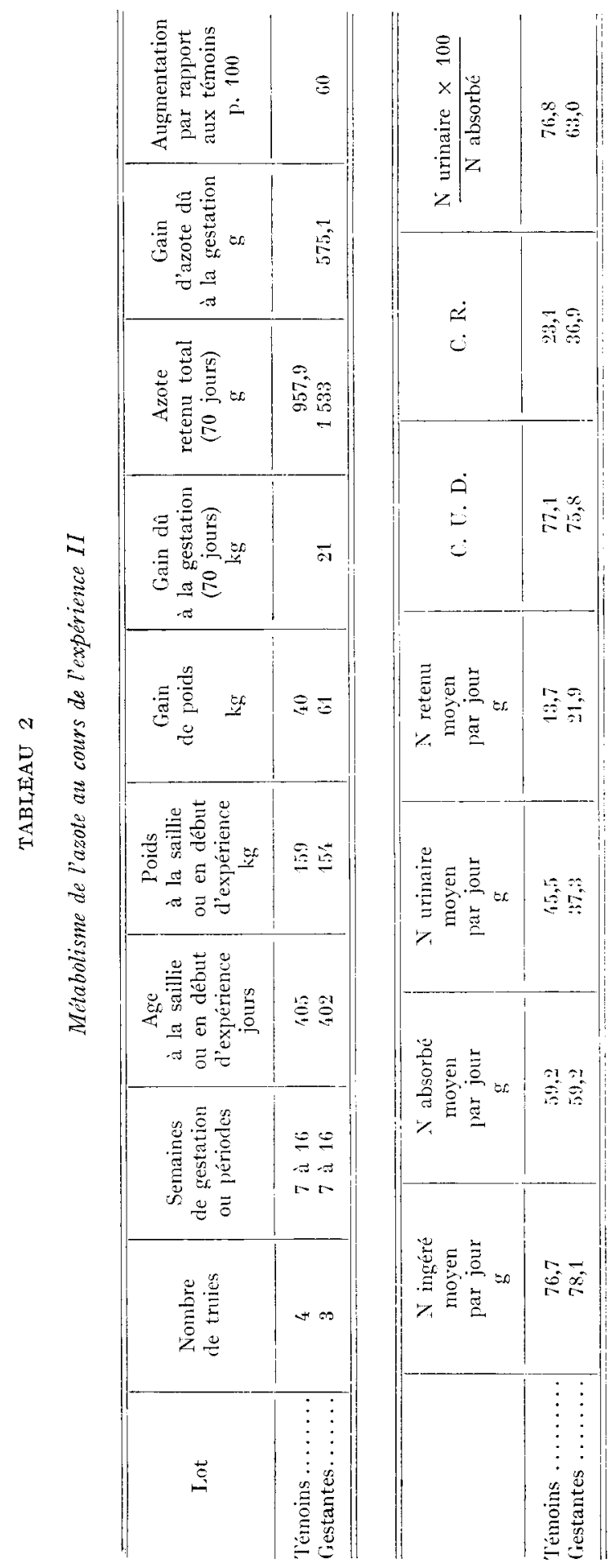


P. ROMBAUTS

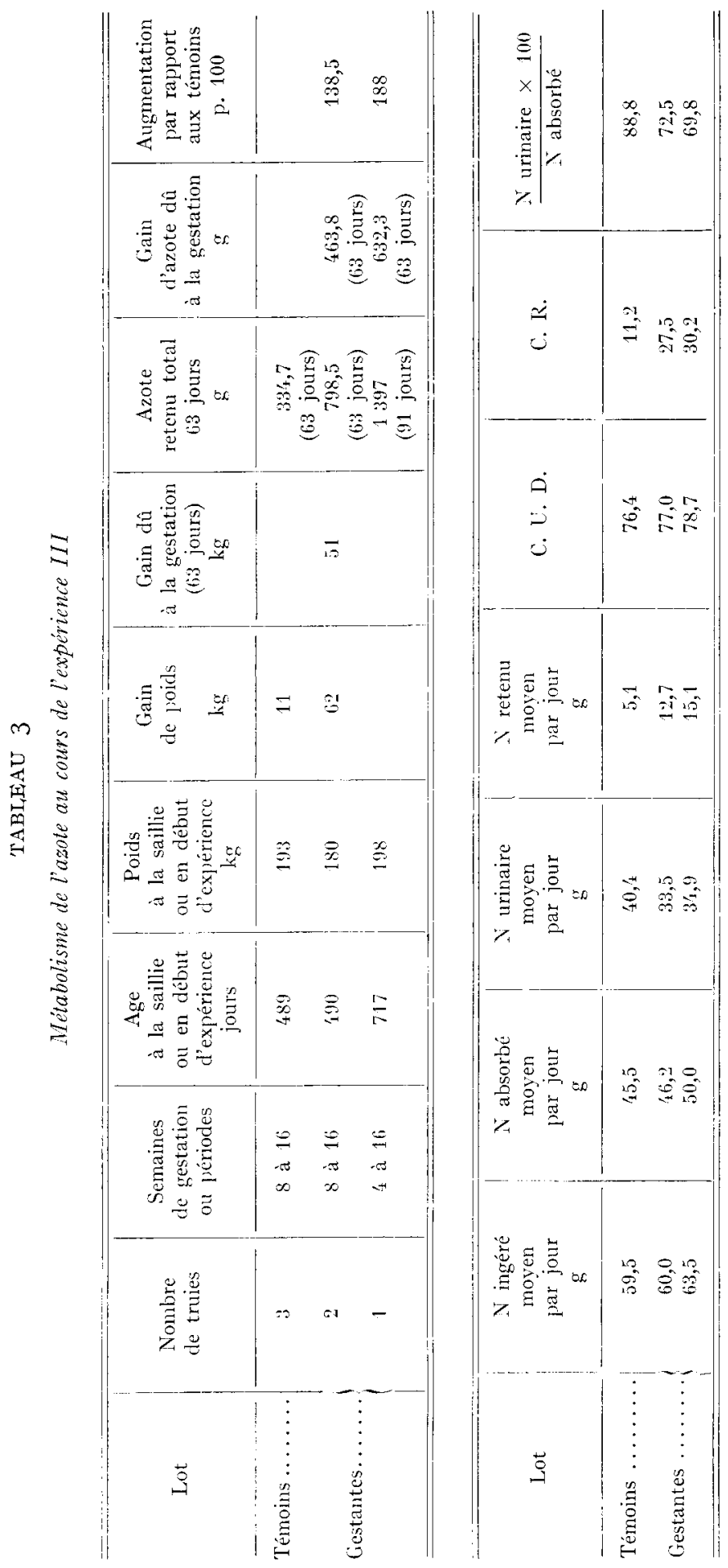




\section{DISCUSSION}

\section{A. - Anabolisme gravidique maternel}

On constate tout d'abord que les besoins d'azote pour l'ensemble des produits de la conception sont toujours largement couverts, même dans le cas de rations limitées - 2,5 kg d'aliment sec — et sans aucun supplément les dernières semaines. Mais pour parler d'anabolisme gravidique maternel il est nécessaire de défalquer des rétentions totales observées, non seulement le contenu utérin, mais les rétentions correspondant aux besoins de croissance et celles dues à la seule influence du niveau alimentaire. L'ensemble de ces rétentions indépendantes de la gravidité est représentée par les rétentions des témoins.

Nous n'avons pas effectué les analyses chimiques des portées, car ces résultats sont actuellement connus.

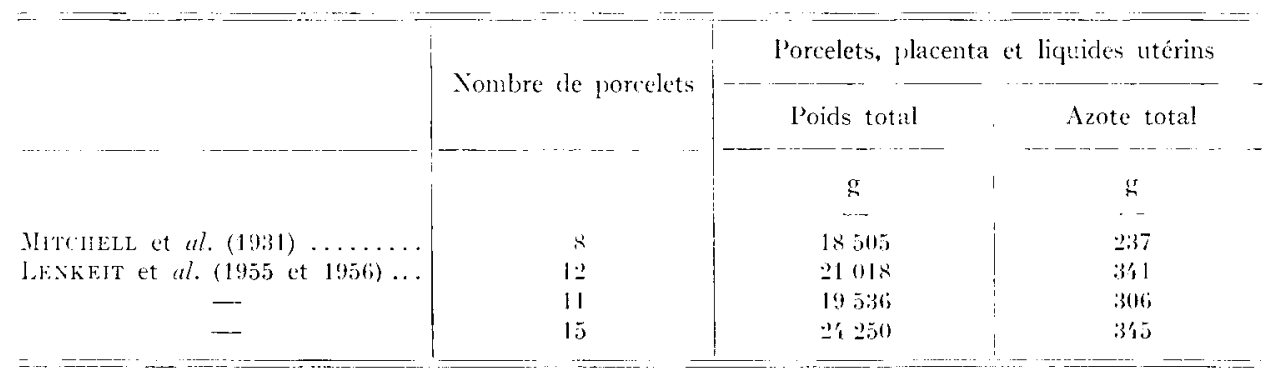

Pour une portée de I 2 porcelets on peut donc adopter les chiffres maxima de 2 I $\mathrm{kg}$ et de $340 \mathrm{~g}$ d'azote.

D'après nos résultats et ceux des auteurs cités précédemment le gain de la truie gravide pour ses propres tissus peut donc " en moyenne " s'évaluer ainsi :

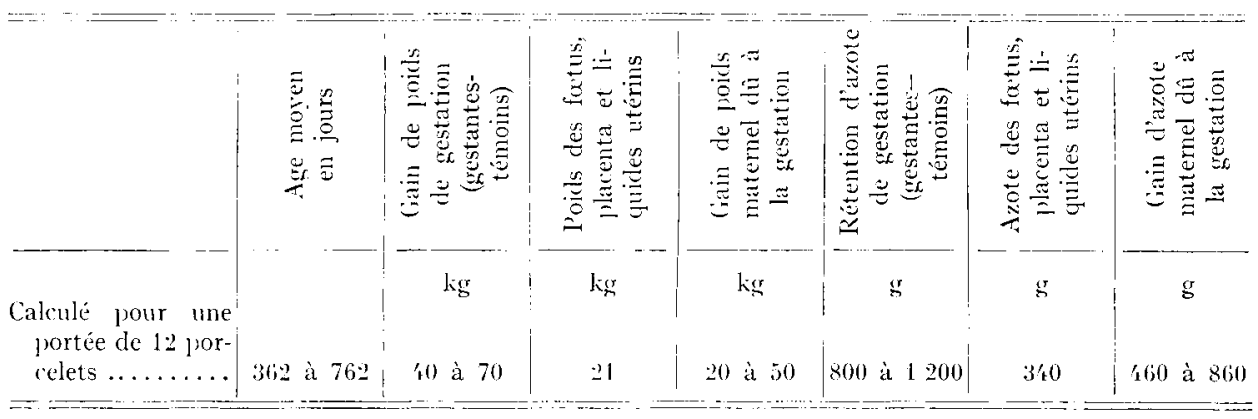

Il est certain qu'avec un niveau alimentaire plus élevé on pourrait obtenir des rétentions encore plus importantes, notamment chez les animaux âgés.

On observe donc toujours une augmentation importante des tissus maternels, en plus de celle des organes reproducteurs, (utérus et mamelles). Ces chiffres sont en accord avec les données publiées par SALMON-LEGAGNEUR et JACQUOT (Ig6I $a$ ) : gain de $30 \mathrm{~kg}$ des carcasses éviscérées par rapport aux témoins. 


\section{B. - Évolution de l'anabolisme au cours de la gravidité}

Chez la 'Truie, mère d'une portée nombreuse, l'anabolisme se manifeste, comme chez le Rat (Rombauts, Bourdei, et Jacquo'T 1956), dès le début de la gestation, alors qu'il n'apparaît que beaucoup plus tardivement chez la Brebis (RomBAUTs I959 $a$ ). Ainsi pendant les 6 premières semaines de gestation de l'expérience I, malgré la croissance élevée des témoins à cet âge $(5 \mathrm{I} 8 \mathrm{~g}$ d'azote retenu) les truies gravides ont retenu $675,8 \mathrm{~g}$ d'azote, soit $30,3 \mathrm{p}$. Ioo de plus que ces témoins (tableau I). A ce stade les gains de poids vif sont d'ailleurs toujours plus élevés que ne le laisseraient prévoir les bilans d'azote car la lipogenèse est accrue pendant cette première partie de la gestation (SALMON-LEGAGNEUR et JACQUOT Ig6I $b$ ).

Mais ces rétentions du début de la gestation sont plus faibles que celles des dernières semaines. 'Toutefois chez des animaux plus âgés l'écart entre gestantes et témoins pourrait être plus important. L'examen des figures 3 et 5 montre, en effet, qu'à partir de la $\mathrm{II}^{\mathrm{e}}$ semaine l'excrétion urinaire d'azote diminue nettement par rapport à l'azote absorbé.

Cette brusque variation du métabolisme apparaît mieux en l'exprimant sous la forme du coefficient d'excrétion métabolique : $\frac{\text { azote urinaire } x}{\text { azote absorbé }}$ roo - Ce coefficient rend parfaitement compte de la rétention apparente au cours d'un état physiologique tel que la gestation pendant laquelle le coefficient de digestibilité ne varie pas; ilest bien entendu qu'il ne faut comparer les coefficients des gestantes qu'à ceux d'animaux de même âge et maintenus qualitativement et quantitativement au même niveau alimentaire (fig. 5 et 6). Ira modification du métabolisme vers la I I $^{\mathrm{e}}$ semaine est particulièrement nette sur la courbe 5. Avec un régime constant (fig. 6), l'excrétion urinaire d'azote s'abaisse progressivement à partir de Io à II semaines alors que l'excrétion des témoins reste en moyenne constante. En début de gestation, en revanche, on n'observe pas de semblable divergence : les deux courbes restent parallèles.

Mais le fait remarquable est que, dès la $3^{\mathrm{e}}$ semaine de gestation, et jusqu'à la II $^{\mathrm{e}}$, il y a toujours un décalage d'environ ro p. Ioo entre les coefficients d'excrétion métabolique des gestantes et ceux des témoins.

On a donc bien un anabolisme gravidique dès le début de la gestation chez la truie, mais cet anabolisme s'accentue brusquement à partir de Io à II semaines.

\section{C. -- L'anabolisme gravidique en fonction de l'age de l'animal}

Les truies, gestantes ou non, étaient soumises au même niveau alimentaire et, à l'exception de quelques semaines de la $2^{\mathrm{e}}$ expérience ce niveau est resté constant. Les quantités totales d'azote retenues varient beaucoup en fonction des ingesta mais par contre, si l'on est assez éloigné de l'équilibre du bilan, les coefficients de rétention ne sont modifiés que pour des variations importantes des quantités ingérées. Ce n'est pas le cas ici, oì dans la $2^{\mathrm{e}}$ expérience les ingesta étaient de $77 \mathrm{~g}$ d'azote au lieu de $60 \mathrm{~g}$ dans les périodes I et 2 qui l'encadrent. On voit sur la figure 7 que la baisse des coefficients de rétention des truies témoins avec l'âge n'a été que peu affectée par la variation du niveau alimentaire au cours de la $2^{\mathbf{e}}$ expérience.

L'examen des 3 expériences successives avec les mêmes témoins, d'âge et de poids égal au départ, permet donc de mettre en évidence un aspect nouveau de l'anabolisme gravidique. L, cs rétentions d'azote excédentaires, dues à la gestation, expri- 
mées en pourcentage des rétentions des témoins passent progressivement, pour la deuxième moitié de la gestation de 60 à $138,5 \mathrm{p}$. I00 quand 1'âge des animaux à la mi-expérience passe de 438 à 52 I jours (tableaux I, 2 et 3 ). Si les rétentions de gravidité diminuent au début avec l'âge elles restent néanmoins toujours très supérieures à celles des truies témoins, même pendant la I re partie de la gestation. Dans le tableau4, nous rapportons les résultats de 2 truies, saillies à l'âge de 542 et 545 jours, donc beaucoup plus âgées que les animaux de l'expérience I. Les rétentions observées sont de 9,5 et 7,4 g d'azote par jour, alors que celles de témoins de même âge peuvent être estimées à moins de 5, I g. Il y a donc bien un anabolisme dès le début de la gravidité, quel que soit l'âge de l'animal.

TABLEAU 4

Frolution de l'anabolisme gravidique avec l'âge de l'animal

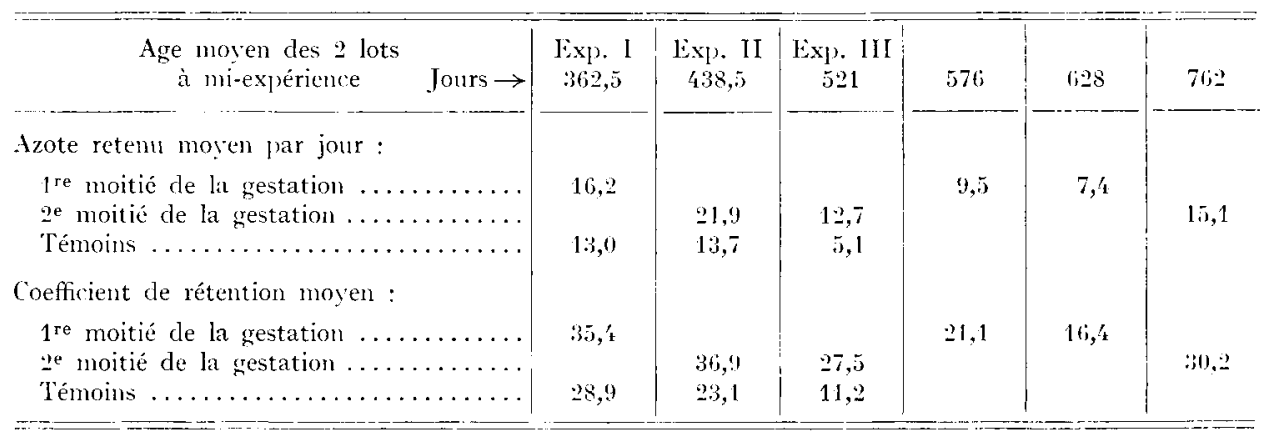

Ceci est encore plus net pendant la $2^{\mathrm{e}}$ moitié de la gestation (tableau 4 , fig. 7 ). Alors que les coefficients de rétention des témoins continuent à baisser avec l'âge, les capacités de rétention des gestantes se maintiennent à un niveau élevé (CR de 27 à 30 p. 100), même chez un animal âgé de 762 jours.

Toutefois on voit que si le gain corporel a été élevé chez les truies gravides pendant l'expérience II - $6 \mathrm{I} \mathrm{kg}$-- les témoins ont pris eux-mêmes $40 \mathrm{~kg}$. On ne peut donc ici parler d'anabolisme maternel de gestation, le gain de poids supplémentaire, $2 \mathrm{I} \mathrm{kg}$, correspondant aux seuls produits de la conception, fœtus et ses annexes. Quand l'animal, pendant la phase de croissance encore active, est déjà à un niveau d'anabolisme élevé, la gestation ne peut augmenter que modérément ses capacités anabolisantes.

Dans l'expérience III, par contre, avec đes animaux âgés de 490 jours à la saillie, et dans nos conditions d'alimentation la croissance est fortement ralentie (tableau 3 ). En 63 jours d'expérience le poids corporel des animaux témoins ne s'est élevé que de I I $\mathrm{kg}$ alors que, pendant la même durée, les truies gravides ont pris $62 \mathrm{~kg}$. La gestation a donc entrainé un gain de poids global de $5 \mathrm{I} \mathrm{kg} \mathrm{et,} \mathrm{après} \mathrm{déduction} \mathrm{des} 2 \mathrm{I} \mathrm{kg}$ que peut représenter le contenu utérin, il reste un gain corporel de $30 \mathrm{~kg}$ pour l'organisme maternel. Le coefficient de rétention moyen des gestantes est de $27,5 \mathrm{p}$. Ioo alors qu'il n'est que de II,2 chez les témoins. Rappelons qu'il s'agit de la I ${ }^{\text {re }}$ gestation de nos animaux. Après une lactation les coefficients de rétention peuvent être sensiblement différents. C'est le cas de la truie saillie à $7 \mathrm{I} 7$ jours dont les résultats ont été 
portés isolément dans le tableau 3. Cette truie avait déjà eu une gestation précédemment et avait allaité sa portée. Le fait que ses rétentions sont légèrement supérieures à celles de sa ${ }^{\text {re }}$ gestation est peut être dû à la lactation précédente, mais, de toute façon, la gestation provoque des rétentions élevées à une époque oì la croissance est faible comme le prouvent les deux autres résultats de cette expérience portant sur une I $^{\text {re }}$ gestation.

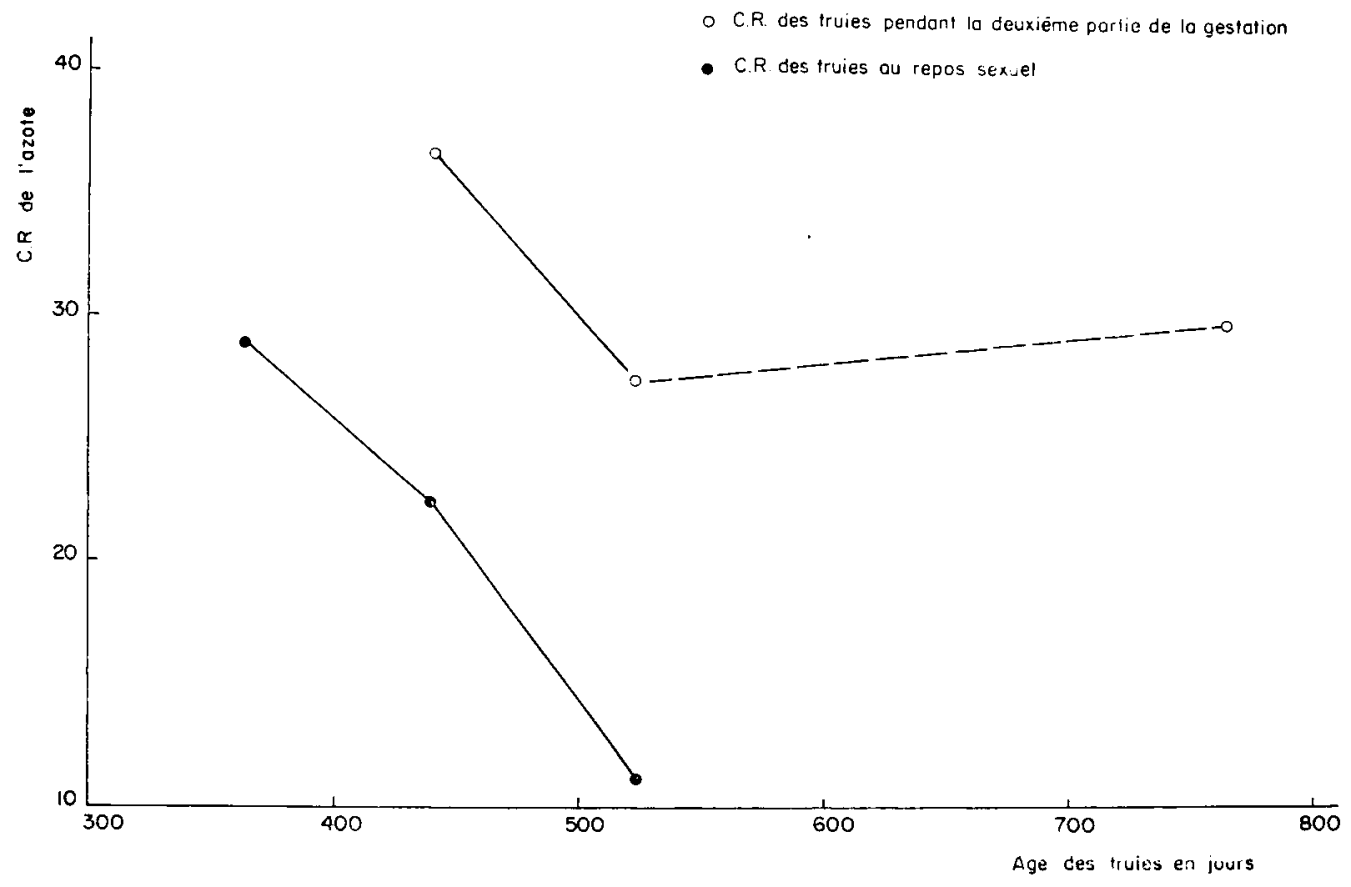

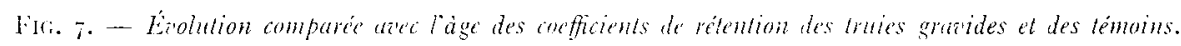

C'est donc chez l'adulte que des gains corporels importants de tissu protéique ne peuvent être attribués qu'à la seule influence de la gravidité. C'est ce que nous avions déjà observé chez la brebis sur des animaux âgés de 5 ans (RombaUts I959 a et b). Le phénomène d'anabolisme gravidique n'en a que plus d'intérêt puisqu'il se mafeste chez un animal adulte qui, en dehors des périodes de reproduction présente un bilan équilibré, quelles que soient les variations de l'apport alimentaire. Le fait que l'anabolisme s'accroisse à partir de Io à I I semaines de gestation ne signifie pas que ce soit la période des gains maternels maxima. A partir de cette époque la croissance des fœtus absorbe en effet une part de plus en plus grande de ces rétentions.

En conclusion, les truies possèdent de remarquables capacités d'utilisation alimentaire au cours de la gestation. L'anabolisme se manifeste dès le début de la gravidité mais s'accentue fortement à partir de la II $^{\mathrm{e}}$ semaine, pendant la période de besoins maxima. La croissance se poursuivant longtemps après la puberté đans cette espèce, on ne peut parler d'anabolisme " maternel de gestation " que chez l'animal en fin de croissance et surtout chez l'adulte. Fitant donné ces fortes capacités de 
rétention d'azote la gestation n'entraînerait que de faibles besoins supplémentaires. La constitution de réserves de gestation, en mettant à profit ces facultés anabolisantes ne se justifie donc que dans la mesure où elles améliorent la production laitière et le bilan maternel de lactation.

$\operatorname{Recu}$ en furior 196:

\section{SUMMARY}

PREGNANCY ANABOLISM IN THE SOW AND ITS RELATIONSHIP TO AGE OF ANIMALS

A study has been made of the nitrogen metabolism in the sow during pregnancy in comparison with that of animals ot the same age and weight at the start of experiment but not gravid.

The pregnant and control animals received qualitatively and quantitatively the same diet in each experiment. The results are given as a function of stage of pregnancy and age of the sows at mating in the firures $I$ to Io and the tables $\mathrm{I}$ to 4.

According to the age of the animals the maternal gains in nitrogen due to pregnancy, when the requirements for glowth and maintenance are deducted, vary from 460 to $860 \mathrm{~g}$ of nitrogen, or is to $30 \mathrm{~kg}$ expressed in muscular tissue, in spite of a relatively restricted feed level, $2,5 \mathrm{~kg}$ dry matter.

Inabolism is seen from the onset of pregnancy but is distinctly accentuated from the I th week. (fig. 7 to 9), the coefficients of retention of nitrogen reaching $30 \mathrm{p}$. 100 during the second half of gestation.

During the stage of active growth following puberty, pregnancy anabolism cannot be termed " matemal " strictly speaking, as the high anabolic level of the animals at this period because of growth, prevents gravidic retentions superior to the needs for the edification of the fuetus, the placenta and uterine liquids.

However it is at the end of growth and in the adult that these anabolising faculties of gestation come fully into force, the nitrogen retentions numbering $\mathrm{I} 3$ to $15 \mathrm{~g}$ per day whilst sows of the same age but not pregnant retain less than $5 \mathrm{~g}$.

\section{RÉFÉRENCES BIBLIOGRAPHIQUES}

Borae A. W., Chanders M. I., Cutmertsos 1). P., 195.3. Gileichgewicht und Verteilung des Stickstoffs bei der trächtigen und laktierenden Ratte. Hoppe Sevler's Ztschr. Physiol. Chem., 295, 424-435.

Enavs R. E., 1929. Protein and mineral metabolism in pregnant sows on a normal or high calcium diet compared with a calcium deficient diet. J. Agric. Sci., 19, 7.52-798.

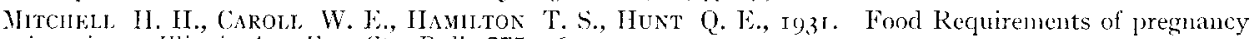
in swine. Ilinois $1 \mathrm{gr}$. Exp. Sta. Bull., 375, 467-504.

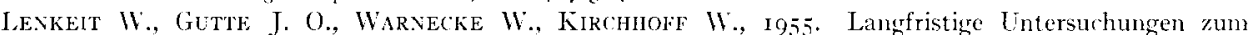
aüseren und inmeren stoffwechsel des gravirlen und laktierenden schweines. Zischr. Tiezern. Fullermillelk, $10,35 \mathrm{r}-364$.

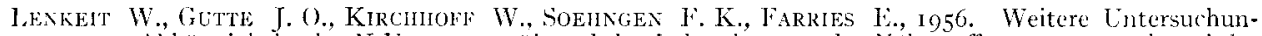
gen zur Abhängigkeit des N-l!msatzes während der Laktation von der Nährstoffversorgung während der inaviditat. 7tschr. Tierem. Fullermillelk., 11, 337-352.

Rombayts P.. Bourdei (i., Jaceuot R., 1956. Analyse de l'anabolisme gravidique che le rat blan en vue de la définition des besoins nutritionnels de la gestation. Arch. Sc. Phi'siol., 10, 173-193.

Rombauts P., i959a. L'Anabolisme de gestation chez la Brebis. C. R. Acad. Sci., 248, 1859-1801.

Rovbal:Ts I’., 1959 b. Étude de l'anabolisme de gestation par l'analyse corporelle des mères. C.R. Soc. Biol., 153, 556-558.

Rombaites I'., г950 c. Anabolisme de gestation et lactation. C. R. Acad. Sci., 248, $2410-2412$.

SALMOX-LEGMixEtR E., JACQCOT R., I96Ia. Modifications corporelles entraînées par lanabolisme gravidique chez li Truic. C: R. Acal. Sci., 253, $54+-546$.

SALMOX-LEGAGETR E., JACQCOT R., I96I $b$. Influence du nival alimentaire sur le comportement nutritionmel de la Truic gestante. C. R. Acad. Sci., 253, i +97-I 499. 